\title{
Improved Time-Series Photometry and Calibration Method for Non-Crowded Fields: MMT Megacam and HAT-South Experiences
}

\author{
Seo-Won Chang ${ }^{1}$, Yong-Ik Byun ${ }^{1}$, and Dae-Won Kim ${ }^{1,2}$ \\ ${ }^{1}$ Department of Astronomy, Yonsei University, Seoul, Korea \\ ${ }^{2}$ Harvard-Smithsonian Center for Astrophysics, Cambridge, MA 02138. USA \\ email: seowony@galaxy.yonsei.ac.kr
}

\begin{abstract}
We present a new photometric reduction method for precise time-series photometry of non-crowded fields that does not need to involve relatively complicated and CPU intensive techniques such as point-spread-function (PSF) fitting or difference image analysis. This method, which combines multi-aperture index photometry and a spatio-temporal de-trending algorithm, gives much superior performance in data recovery and light-curve precision. In practice, the brutal filtering that is often applied to remove outlying data points can result in the loss of vital data, with seriously negative impacts on short-term variations such as flares. Our method utilizes nearly $100 \%$ of available data and reduces the rms scatter to several times smaller than that for archived light curves for brighter stars. We outline the details of our new method, and apply it to cases of sample data from the MMT survey of the M37 field, and the HAT-South survey.
\end{abstract}

Keywords. methods: data analysis - techniques: photometric

\section{Multi-Aperture Index Photometry}

Our photometric technique is similar to standard aperture photometry, except that we compute the flux in a sequence of several apertures and then determine the optimal aperture (with maximum $\mathrm{S} / \mathrm{N}$ ) individually for each object at each epoch. We also add a new index that isolates peculiar situations where photometry returns misleading information. The method is as follows:

(a) Build the master astrometric catalogue. Since the relative centroid positions of all objects are the same for all frames in the time-series, we can easily place an aperture and measure the flux even for the fainter stars.

(b) Refine the centroid position by the Gaussian window. The precision in centroiding is very close to that of PSF-fitting on properly sampled point sources.

(c) Perform photometry with multiple apertures, then find the optimal aperture size by measuring the $\mathrm{S} / \mathrm{N}$ values for each object. We achieve the optimal balance between flux loss and noise based on the revised CCD equation (Howell \& Everett 2001). For sky background, the annulus value is calculated by a combination of $\kappa-\sigma$ clipping and mode estimation. We also use a background map to reduce contamination from nearby sources.

(d) Decide the final aperture with our indexing method (Fig. 1). Differential magnitudes between apertures are compared with mean trends for stars of similar brightness, and the difference is indexed. When a signature of contamination occurs, different apertures and an aperture correction term are automatically applied. In that way we can measure correct fluxes even for contaminated stars. 


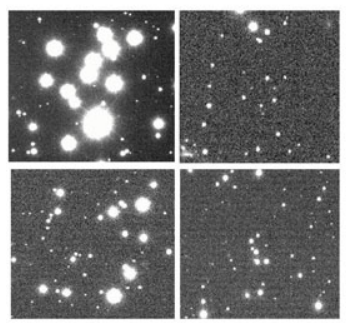

Grouping the stars on the basis of raw or transformed magnitude

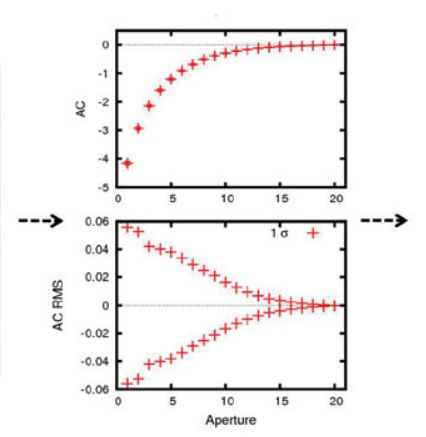

Modeling the RMS of aperture correction terms in each group

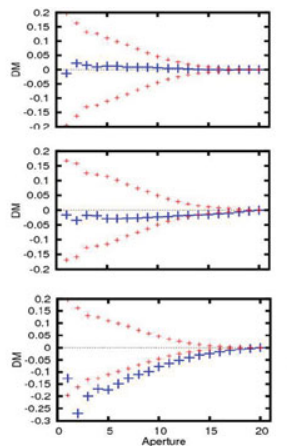

No

contamination

Contamination @larger aperture

Contamination @smaller aperture

Indexing the contaminated aperture using differential magnitude data $\left(=\mathrm{AP}_{\text {ref }}-\mathrm{AP}_{\mathrm{i}}\right)$ for each object

Figure 1. Sequence of multi-aperture index photometry. We can see whether, and at what aperture, the differential magnitude begins to diverge from the model curve for each epoch.

\section{Spatio-Temporal Photometric De-trending}

In order to reduce systematic effects in time-series photometry, several successful methods have been introduced (e.g., Sys-Rem: Tamuz et al. 2005; TFA: Kovacs et al. 2005; PDT: Kim et al. 2009). Among those, we find that PDT is the most useful for detecting and removing spatially localized patterns in time-series data, as trends in our sample data do show a tendency of localization. Why the trends are different and localized within a single CCD frame is a subject for further study, but is probably related to subtle changes in the PSF and sky conditions within the detector field of view. We apply the PDT algorithm in following steps:

(a) Select the template light curves for bright stars. Within the observation span, these light curves have the same temporal ordering of data points. We take a sequence of data points, $L_{i}\left(t_{r e f}\right)$, as the reference time line.

(b) Extract all subset of light curves that show spatially and temporally correlated features such as clusters. The strength of linear dependence between two or more light curves is expressed as the Pearson correlation coefficient. Each cluster is determined by an hierarchical tree clustering algorithm.

(c) Construct master trends for each cluster, $T_{c}\left(t_{r e f}\right)$. Master trends are obtained as the weighted averages of normalized differential light curves, $f_{i}\left(t_{r e f}\right)$, as described below:

$$
T_{c}\left(t_{r e f}\right)=\frac{\sum_{i=1}^{N_{c}} \omega_{i} f_{i}\left(t_{r e f}\right)}{\sum_{i=1}^{N_{c}} \omega_{i}}, f_{i}\left(t_{r e f}\right)=\frac{L_{i}\left(t_{r e f}\right)-\bar{L}_{i}}{\bar{L}_{i}}, \omega_{i}=\frac{1}{\sigma_{f_{i}}^{2}}
$$

(d) De-trend the light curves of all stars with matching master trend and time lines: we adjust trend sets for each light curve, $f_{i}\left(t_{i}\right)$, and then determine free parameters $\left(\beta_{i c}\right)$ by minimizing the noise term $\left(\epsilon_{i}\right)$.

$$
f_{i}\left(t_{i}\right)=\sum_{c} \beta_{i c} T_{c}\left(t_{i}\right)+\epsilon_{i}\left(t_{i}\right)
$$



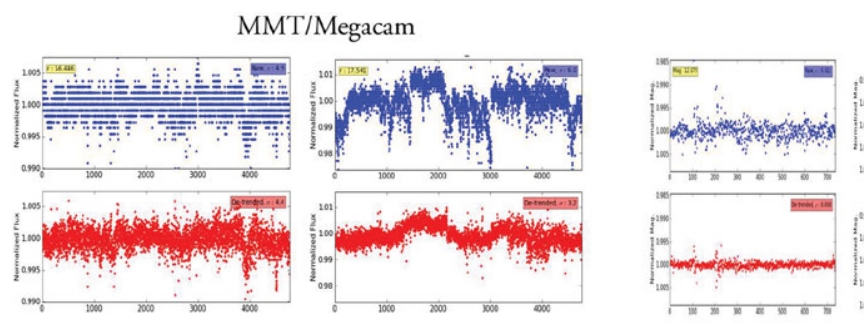

HAT-South
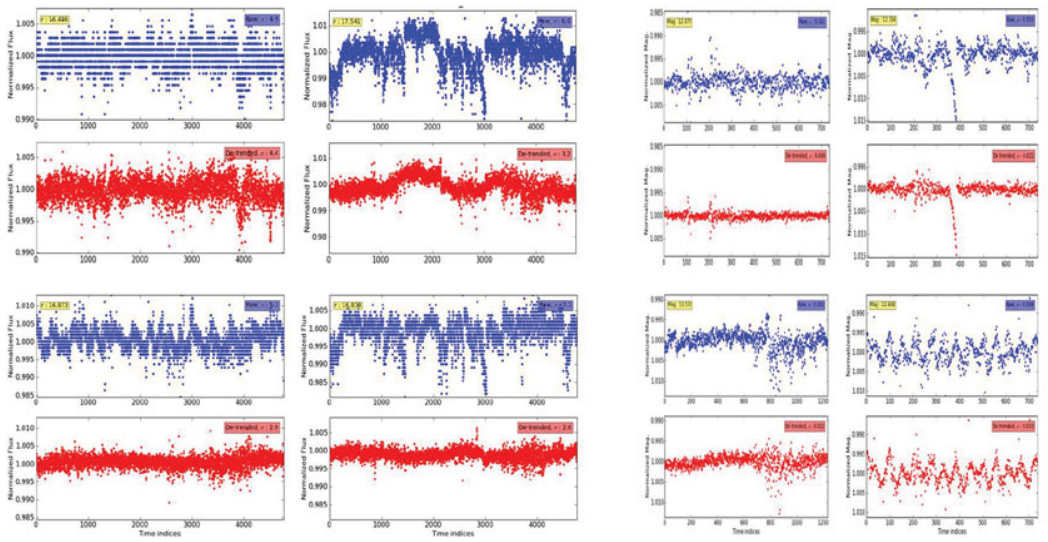
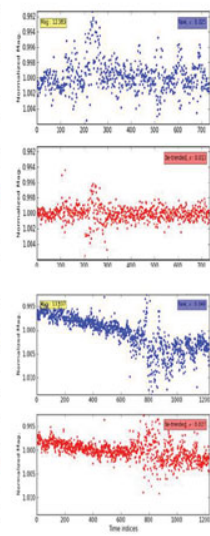

Figure 2. In each subset of MMT/megacam and HAT-South data a multi-aperture indexed light curve (above) is paired with the de-trended light curve (below).

\section{Experiments with Time-Series Data}

Time-series samples were obtained from two different types of transit survey projects: (a) one month of MMT M37 survey data, using the Megacam instrument $(362 \mathrm{k} \times 4 \mathrm{k}$ CCDs, $24^{\prime} \times 24^{\prime}$ field of view) (Hartman et al. 2008), (b) HAT-South survey data, from a network of wide-field (one camera $\sim 4^{\circ} .2 \times 4^{\circ} .2$ field of view) telescopes in Australia, Chile and Namibia (Bakos et al. 2009). Fig. 2 shows examples of light curves that demonstrate the effect of our de-trending method. Some systematic effects are clearly present in the light curves of bright stars, yielding artificial flux variations. Our method removes only the systematic variations that are shared by light curves of stars in the adjacent sky regions; all kinds of true variability are preserved.

\section{Acknowledgements}

This work is supported by the Korea Institute of Science and Technology Information under the contract of the commissioned research project, Massive Astronomical Data Applications of Cloud Computation (KISTI-P11020). We thank Joel D. Hartman and Matthew J. Holman for their suggestion to use the whole MMT data set. We also thank Daniel Bayliss and the HAT-South team for helping us access HAT-South data.

\section{References}

Bakos, G., et al. 2009, in: F. Pont, D. Saeelov, \& M. Holman (eds.), Transiting Planets, IAU Symposium No. 253 (Cambridge: Cambridge Univ. Press), p. 354

Hartman, J. D., et al. 2008, ApJ, 675, 1233

Howell, S. B. \& Everett, M. E. 2001, in: W.J. Borucki \& L.E. Lasher (eds.), 3rd Workshop on Photometry, p. 1

Kim, D.-W., Protopapas, P., Alcock, C., Byun, Y.-I., \& Bianco, F. B. 2009, MNRAS, 397, 558

Kovács, G., Bakos, G., \& Noyes, R. W. 2005, MNRAS, 356, 557

Tamuz, O., Mazeh, T., \& Zucker, S. 2005, MNRAS, 356, 1466 